\title{
ANALYSIS AND DESIGN OF SOFTLY SWITCHED MODEL PREDICTIVE CONTROL
}

\author{
Jingsong Wang ${ }^{(1)}$, Michal Grochowski ${ }^{(2)}$, Mietek A. Brdys ${ }^{(1)}$ \\ ${ }^{(1)}$ School of Electronic, Electrical and Computer Engineering \\ The University of Birmingham,Birmingham B15 2TT,UK.Email: m.brdys@bham.ac.uk \\ ${ }^{(2)}$ Faculty of Electrical and Control Engineering, Gdansk University of Technology \\ Narutowicza11/12 80-952 Gdansk, Poland.Email:mgrochow@ely.pg.gda.pl
}

\begin{abstract}
This paper presents a new approach to soft switching between two model predictive controllers. The motivation for this work comes from the control of large scale hierarchical systems where different operating scenario asking for different control objective makes a single model predictive controller (MPC) unsuitable. The proposed soft switching approach shows much better switching performance both in system output and control input than the traditional hard switching. The stability of the designed soft switching process is analysed and sufficient conditions for stability are derived. Numerical examples with simulation results show that the proposed approach can be useful in practical applications. Copyright (C) 2005 IFAC
\end{abstract}

Keywords: predictive control, discrete-time systems, constraints, stability analysis, supervisory control, sequential switching

\section{INTRODUCTION}

Model-based predictive control, or receding horizon control, has become a very popular controller design method in the process industry. It refers to a class of algorithms where an optimization problem is solved repetitively at every new time step. Only the first element of the computed control sequence is applied to the plant. Notable past reviews of MPC include those of (Muske and Rawlings, 1993; Mayne, et al., 2000; Qin and Badgwell, 2003).

With the increasing use of MPC into large scale and complex hierarchical systems, the demand to use multiple MPCs to meet different control objectives under different operating scenarios arises (Grochowski, et al., 2004a). The so called softly switched MPC (SS-MPC) means such an approach that can make the switch between two MPCs smooth by contrast with hard switching whose switching performance is often unsatisfactory especially in the scenarios where the input/state constraints are changing from very tight constraints to much looser ones. A real life application can be found in (Grochowski, et al., 2004b). Although the issues of stability of constrained model predictive control for linear time-invariant (LTI) systems have been deeply investigated (Zheng and Morari, 1995; Mayne, et al., 2000; Primbs and Nevistic, 2000), the research on the stability of switching among multiple constrained predictive controllers is still an open area which incorporates fundamental problems both in the MPC area and in the switched system field (Grochowski, 2003). In (Zheng, 1997), the issue of time-varying weights in model predictive control has been discussed, but it was limited to varying weights only inside the prediction horizon and the weights between each new iterate time step remain the same. In this paper, a softly switched MPC (SS-MPC) method is proposed to deal with a more general and complicated problem than the one put forward by Zheng (1997), that is, the weights change not only within the prediction horizon but also at every new iterate time step.

This paper is organised as follows: the problem is formulated in Section 2; a soft switching mechanism with two specific methods is presented in Section 3; stability issue of the proposed methods is discussed in Section 4; a numerical example to test the effectiveness of the proposed methods is shown in Section 5; finally, conclusions are made in Section 6. 


\section{PROBLEM FORMULATION}

Consider a discrete linear time-invariant system:

$$
\left\{\begin{array}{l}
x(k+1)=A x(k)+B u(k) \\
y(k)=C x(k)
\end{array}\right.
$$

with $A \in \mathbb{R}^{n \times n}, B \in \mathbb{R}^{n \times m} . \quad x(k) \in \mathbb{R}^{n}$ and $u(k) \in \mathbb{R}^{m}$ denote the state and control input at time $k$ respectively. The system (1) has the following constraints on control inputs:

$$
u(k) \in \mathbf{U} \subseteq \mathbb{R}^{m}
$$

where $\mathbf{U}$ is convex sets containing the origin in their interior.

The finite prediction horizon MPC formulation is characterized by the online minimization of the following performance index with a terminal cost subject to system equation (1) and the constraints:

$$
\begin{aligned}
\min _{u(\cdot k)} J_{N}(x(k), u(\cdot \mid k)) \\
=\min _{u(\cdot \mid k)} \sum_{i=0}^{N-1}\left[\begin{array}{l}
x^{T}(k+i \mid k) Q x(k+i \mid k) \\
+u^{T}(k+i \mid k) R u(k+i \mid k)
\end{array}\right] \\
+x^{T}(k+N \mid k) P x(k+N \mid k)
\end{aligned}
$$

subject to: $x(k \mid k)=x(k)$

$$
\begin{aligned}
& x(k+i+1 \mid k)=A x(k+i \mid k)+B u(k+i \mid k) \\
& u(k+i \mid k) \in \mathbf{U} \subseteq \mathbb{R}^{m} \quad \forall i \in\{0, \cdots, N-1\}
\end{aligned}
$$

where $\mathrm{Q}, \mathrm{P}$ are positive semi-definite matrices, $\mathrm{R}$ is positive definite matrix and $x(\cdot \mid k)$ and $u(\cdot \mid k)$ are the predicted state and control sequences at time $k$.

The receding horizon control based on problem (3) is regarded as an MPC, which is determined by N, Q, R, $\mathrm{P}$ and $\mathbf{U}$. In real applications, switch from one MPC to another one in a controlled satisfactory way is a challenging problem faced by the process control industry. More specifically, how to switch from the old MPC, which is

$$
\begin{aligned}
\min _{u(\cdot \mid k)} J_{N_{1}}(x(k), u(\cdot \mid k)) \\
=\min _{u(\cdot \mid k)} \sum_{i=0}^{N_{1}-1}\left[\begin{array}{l}
x^{T}(k+i \mid k) Q_{1} x(k+i \mid k) \\
+u^{T}(k+i \mid k) R_{1} u(k+i \mid k)
\end{array}\right] \\
+x^{T}(k+N \mid k) P_{1} x(k+N \mid k)
\end{aligned}
$$

subject to: $x(k \mid k)=x(k)$

$$
\begin{aligned}
& x(k+i+1 \mid k)=A x(k+i \mid k)+B u(k+i \mid k) \\
& u(k+i \mid k) \in \mathbf{U}_{1} \subseteq \mathbb{R}^{m} \quad \forall i \in\{0, \cdots, N-1\}
\end{aligned}
$$

to the new MPC, which is

$$
\begin{aligned}
\min _{u(\cdot \mid k)} J_{N_{2}}(x(k), u(\cdot \mid k)) \\
=\min _{u(\cdot k)} \sum_{i=0}^{N_{2}-1}\left[\begin{array}{l}
x^{T}(k+i \mid k) Q_{2} x(k+i \mid k) \\
+u^{T}(k+i \mid k) R_{2} u(k+i \mid k)
\end{array}\right] \\
\quad+x^{T}(k+N \mid k) P_{2} x(k+N \mid k)
\end{aligned}
$$

subject to: $x(k \mid k)=x(k)$

$$
\begin{aligned}
& x(k+i+1 \mid k)=A x(k+i \mid k)+B u(k+i \mid k) \\
& u(k+i \mid k) \in \mathbf{U}_{2} \subseteq \mathbb{R}^{m} \quad \forall i \in\{0, \cdots, N-1\}
\end{aligned}
$$

is the main problem to be dealt with in this paper.
In the following sections, the old MPC and the new MPC are assumed, for simplicity, to have the same length of prediction horizon as $N \triangleq N_{1}=N_{2}$.

\section{SOFT SWITCHING MECHANISMS}

The most direct way to switch from the old MPC (4) to the new MPC (5) is by hard switching without any intermediate switching process. Although such hard switching is very simple, it does not usually give satisfactory switching outcome because the switching is in such a sudden way that it can probably cause some unexpected impulsive phenomena such as huge peak overshoot of state/output, abrupt change of control input and even actuator failure if the control objectives of the two MPCs greatly differ. Such unwelcome switching process is very likely to occur especially under the scenarios where system constraints are changed from very tight constraints to relatively loose ones. Therefore, it is necessary to consider a soft switching mechanism to make the switching process smooth. In this section, following (Grochowski, 2003), two soft switching methods are presented in details.

\subsection{Convex Combined Method}

In this method of SS-MPC, a soft switching process will be designed between the old MPC and the new MPC. During the soft switching process, a number of intermediate MPCs called combined MPCs are introduced. Each combined MPC has the following performance index (6) which is composed by convex combination of the performance indices of the old MPC and the new MPC, i.e. (4a) and (5a).

$$
\begin{aligned}
& J_{\text {ConvexCombined }}(x(k), u(\cdot \mid k)) \\
& =\sum_{i=0}^{N-1}\left[\begin{array}{l}
x^{T}(k+i \mid k)\left[(1-\alpha) Q_{1}+\alpha Q_{2}\right] x(k+i \mid k) \\
+u^{T}(k+i \mid k)\left[(1-\alpha) R_{1}+\alpha R_{2}\right] u(k+i \mid k)
\end{array}\right] \\
& \quad+x^{T}(k+N \mid k)\left[(1-\alpha) P_{1}+\alpha P_{2}\right] x(k+N \mid k)
\end{aligned}
$$

where $\alpha$ is a tuning knob which changes increasingly from 0 to 1 along time axis during the switching process. A specific value of $\alpha$ determines a specific combined MPC.

When $0<\alpha<1$, the combined MPC reads:

$$
\min _{u(\cdot \mid k)} J_{\text {ConvexCombined }}(x(k), u(\cdot \mid k))
$$

subject to: $x(k \mid k)=x(k)$

$$
\begin{aligned}
& x(k+i+1 \mid k)=A x(k+i \mid k)+B u(k+i \mid k) \\
& u(k+i \mid k) \in \mathbf{U}_{2} \subseteq \mathbb{R}^{m} \quad \forall i \in\{0, \cdots, N-1\}
\end{aligned}
$$

When $\alpha=1$, the combined MPC becomes the new MPC and the soft switching process terminates. At each new time step, a different value between 0 and 1 is assigned to $\alpha$. The switching speed fully depends on the changing rate of $\alpha$. 


\subsection{General Combined Method}

The Convex Combined Method shown above has the merits of easy to understand and simple to apply, but it gives the same weights to all the predicted state within the prediction horizon. Here, a dedicated soft switching method which resorts to a more flexible construction of the combined MPC is proposed. The corresponding performance index of the general combined MPC is defined as:

$$
\begin{aligned}
& J_{\text {GeneralCombined }}(x(k), u(\cdot \mid k))= \\
& \sum_{i=0}^{N-1}\left[\begin{array}{l}
x^{T}(k+i \mid k)\left[w_{1}^{k}(i) Q_{1}+w_{2}^{k}(i) Q_{2}\right] x(k+i \mid k) \\
+u^{T}(k+i \mid k)\left[w_{1}^{k}(i) R_{1}+w_{2}^{k}(i) R_{2}\right] u(k+i \mid k)
\end{array}\right] \\
& +x^{T}(k+N \mid k)\left[w_{1}^{k}(N) P_{1}+w_{2}^{k}(N) P_{2}\right] x(k+N \mid k)
\end{aligned}
$$

where $w_{1}^{k}$ and $w_{2}^{k}$ are called weighting vectors whose elements are not only different over the prediction horizon but also vary with time steps.

As the switching process progresses all the elements of the weighting vector $w_{1}^{k}$ decrease to 0 and adversely, all the elements of the weighting vector $w_{2}^{k}$ increase to 1 . This represents the soft switching process from the old MPC to the new MPC. Time at which the new MPC is fully in place is called switched time and this is another, apart from the weighting vectors, important parameter of the soft switching mechanism. Its design is beyond scope of this paper. Hence, in order to simplify notations we shall assume that the switching process terminates after $N$ time steps.

Actually, at each new time step during the switching process, a different combined MPC will be applied to the plant:

$$
\min _{u(\cdot \mid k)} J_{\text {GeneralCombined }}(x(k), u(\cdot \mid k))
$$

subject to: $x(k \mid k)=x(k)$

$$
\begin{aligned}
& x(k+i+1 \mid k)=A x(k+i \mid k)+B u(k+i \mid k) \\
& u(k+i \mid k) \in \mathbf{U}_{2} \subseteq \mathbb{R}^{m} \forall i \in\{0, \cdots, N-1\}
\end{aligned}
$$

Let the switching starts at $k=k_{s}$. The values of $w_{1}^{k}(i)$ and $w_{2}^{k}(i)$ can be determined by the following algorithm:

Algorithm 1.

- If $k+i<N+k_{s}, w_{1}^{k}(i)=\lambda^{k-k_{s}+i}, i=0, \cdots, N-1$

- If $k+i \geq N+k_{s}, w_{1}^{k}(i)=0, i=0, \cdots, N-1$

- $w_{1}^{k}(N)=w_{1}^{k}(N-1)$

- $w_{2}^{k}(i)=1-w_{1}^{k}(i) \quad i=0, \cdots, N$

where $0 \leq \lambda \leq 1$ is a tuning knob. If letting $\lambda=0$, the above algorithm can represent hard switching.

For example, let prediction horizon $N=9, \lambda=0.5$, and $k_{s}=100$ then the weighting vectors $w_{1}^{k}$ and $w_{2}^{k}$ are listed in sequence as follows.
At time step $k=100$, the switching process starts:

$$
\begin{aligned}
& w_{1}^{100}=\left[1\left(\frac{1}{2}\right)^{1}\left(\frac{1}{2}\right)^{2}\left(\frac{1}{2}\right)^{3}\left(\frac{1}{2}\right)^{4}\left(\frac{1}{2}\right)^{5}\left(\frac{1}{2}\right)^{6}\left(\frac{1}{2}\right)^{7}\left(\frac{1}{2}\right)^{8}:\left(\frac{1}{2}\right)^{8}\right] \\
& w_{2}^{100}(i)=1-w_{1}^{100}(i) \quad i=0, \cdots, N
\end{aligned}
$$

At time step $k=101$ :

$$
\begin{array}{ll}
w_{1}^{101}=\left[\left(\frac{1}{2}\right)^{1}\left(\frac{1}{2}\right)^{2}\left(\frac{1}{2}\right)^{3}\left(\frac{1}{2}\right)^{4}\left(\frac{1}{2}\right)^{5}\left(\frac{1}{2}\right)^{6}\left(\frac{1}{2}\right)^{7}\left(\frac{1}{2}\right)^{8} 00 \vdots 0\right] & \\
w_{2}^{101}(i)=1-w_{1}^{101}(i) & i=0, \cdots, N
\end{array}
$$

At time step $k=102$ :

$$
\begin{aligned}
& w_{1}^{102}=\left[\left(\frac{1}{2}\right)^{2}\left(\frac{1}{2}\right)^{3}\left(\frac{1}{2}\right)^{4}\left(\frac{1}{2}\right)^{5}\left(\frac{1}{2}\right)^{6}\left(\frac{1}{2}\right)^{7}\left(\frac{1}{2}\right)^{8} \quad 0 \quad 0 \quad \vdots 0\right] \\
& w_{2}^{102}(i)=1-w_{1}^{102}(i) \quad i=0, \cdots, N
\end{aligned}
$$

At time step $k=109$, the switching process ends:

$$
\begin{aligned}
& w_{1}^{109}=\left[\begin{array}{llllllllll}
0 & 0 & 0 & 0 & 0 & 0 & 0 & 0 & 0 & \vdots
\end{array}\right] \\
& w_{2}^{109}(i)=1-w_{1}^{109}(i) \quad i=0, \cdots, N
\end{aligned}
$$

The soft switching process ends after 9 control steps and the new MPC is now in place after 10 switches have been performed. The algorithm "shifts leftward one bit" the weighting vectors when time step $k$ increases by one. The duration time of the soft switching process is fully controllable. For example, it is easy to speed up the switching process by just shifting leftward two bits or even three bits.

\section{STABILITY OF SS-MPC}

In this section, stability of the above proposed softly switched MPC is investigated. It is assumed that the corresponding optimization problems always have a feasible solution.

Sufficient conditions for stabilizing the finite horizon MPC with terminal cost have been discussed in (Bloemen, et al., 2002), where the inequality relation between the terminal weighting matrix and the linear static state feedback law has been established. Here, a simple sufficient condition for stability of single MPC without local linear static state feedback law is derived first to be the base for further discussions on stability of SS-MPC.

Lemma 1. If $P-A^{T} P A^{T}-Q>0$, then asymptotic stability of the closed-loop system with the MPC (3) can be guaranteed.

Proof. A common approach to stability analysis for receding horizon control is to consider the optimal value of $J_{N}(x(k), u(\cdot \mid k))$ as a candidate Lyapunov function (Primbs and Nevistic, 2000). That is,

$$
V(x(k))=\min _{u(\cdot k)} J_{N}(x(k), u(\cdot \mid k)) .
$$

$V(x(k))$ can be proved to be a Lyapunov function by comparing the optimal cost at time step $k$ with the non-optimal cost at $k+1$ attained by copying the last $N-1$ control moves in $V(x(k))$ plus a zero control move. Such control sequence is feasible over time period $\overline{k+1: k+1+N}$ because there are no state constraints and zero is an admissible control value. Indeed, the following holds: 


$$
\begin{aligned}
& V(x(k)-V(x(k+1)) \\
& \geq x^{T}(k \mid k) Q x(k \mid k)+u_{\text {opt }}^{T}(k \mid k) Q u_{\text {opt }}(k \mid k) \\
& \quad+x^{T}(k+N \mid k) P x(k+N \mid k) \\
& \quad-x^{T}(k+N \mid k) Q x(k+N \mid k) \\
& \quad-x^{T}(k+N \mid k) A^{T} P A x(k+N \mid k)
\end{aligned}
$$

If $P-A^{T} P A^{T}-Q>0$ then $V(x(k)-V(x(k+1))>0$.

Proposition 1. If controller parameters of the old and the new MPC can satisfy:

$$
P_{1}-A^{T} P_{1} A-Q_{1}>0 \text { and } P_{2}-A^{T} P_{2} A-Q_{2}>0,
$$

then asymptotic stability of the closed-loop system with the convex combined MPC (7) can be guaranteed.

Proof. From Lemma 1, the closed-loop system with the combined MPC (7) is asymptotically stable if the following inequality holds:

$$
\begin{aligned}
& {\left[(1-\alpha) P_{1}+\alpha P_{2}\right]-A^{T}\left[(1-\alpha) P_{1}+\alpha P_{2}\right] A } \\
&-\left[(1-\alpha) Q_{1}+\alpha Q_{2}\right]>0
\end{aligned}
$$

This clearly holds when the conditions in Proposition 1 are satisfied.

In order to analyse stability of every single general combined MPC, $w_{1}^{k}$ and $w_{2}^{k}$ are denoted as $w_{1}$ and $w_{2}$ to ignore the effect of $k$ on the weighting vectors.

Proposition 2. Assume the following holds:

- $P_{1}-A^{T} P_{1} A-Q_{1}>0, P_{2}-A^{T} P_{2} A-Q_{2}>0$

- $\Delta Q_{1}(i)+\Delta Q_{2}(i) \geq 0, \Delta R_{1}(i)+\Delta R_{2}(i) \geq 0$

- $w_{1}(N) \geq w_{1}(N-1), w_{2}(N) \geq w_{2}(N-1)$,

where $\quad \Delta Q_{1}(i)=\left[w_{1}(i)-w_{1}(i-1)\right] Q_{1}$

$$
\begin{aligned}
& \Delta Q_{2}(i)=\left[w_{2}(i)-w_{2}(i-1)\right] Q_{2} \\
& \Delta R_{1}(i)=\left[w_{1}(i)-w_{1}(i-1)\right] R_{1} \\
& \Delta R_{2}(i)=\left[w_{2}(i)-w_{2}(i-1)\right] R_{2}
\end{aligned}
$$

Then asymptotic stability of the closed-loop system can be guaranteed for each general combined MPC.

Proof. Let $\psi_{x}(i)=w_{1}(i) Q_{1}+w_{2}(i) Q_{2} \quad i=0, \cdots, N-1$

$$
\begin{aligned}
& \psi_{u}(i)=w_{1}(i) R_{1}+w_{2}(i) R_{2} \quad i=0, \cdots, N-1 \\
& \psi_{x}(N)=w_{1}(N) P_{1}+w_{2}(N) P_{2}
\end{aligned}
$$

The combined MPC reads:

$$
\begin{aligned}
\min _{u(\cdot \mid k)} J_{N}(x(k), u(\cdot \mid k)) \\
=\min _{u(\cdot k)} \sum_{i=0}^{N-1}\left[\begin{array}{l}
x^{T}(k+i \mid k) \psi_{x}(i) x(k+i \mid k) \\
+u^{T}(k+i \mid k) \psi_{u}(i) u(k+i \mid k)
\end{array}\right] \\
+x^{T}(k+N \mid k) \psi_{x}(N) x(k+N \mid k)
\end{aligned}
$$

subject to: $\quad x(k \mid k)=x(k)$

$$
\begin{aligned}
& x(k+i+1 \mid k)=A x(k+i \mid k)+B u(k+i \mid k) \\
& u(k+i \mid k) \in \mathbf{U}_{2} \subseteq \mathbb{R}^{m} \quad \forall i \in\{0, \cdots, N-1\}
\end{aligned}
$$

Using the similar arguments as in proof of Lemma 1, the following inequality can be obtained:

$$
\begin{aligned}
& V(x(k))-V(x(k+1)) \\
& \geq x^{T}(k \mid k) \psi_{x}(0) x(k \mid k)+u_{o p t}^{T}(k \mid k) \psi_{u}(0) u_{o p t}(k \mid k)
\end{aligned}
$$

$$
\begin{aligned}
& +\sum_{i=1}^{N-1}\left[\begin{array}{l}
x^{T}(k+i \mid k)\left(\psi_{x}(i)-\psi_{x}(i-1)\right) x(k+i \mid k)+ \\
u_{o p t}^{T}(k+i \mid k)\left(\psi_{u}(i)-\psi_{u}(i-1)\right) u_{o p t}(k+i \mid k)
\end{array}\right] \\
& +x^{T}(k+N \mid k)\left[\begin{array}{l}
\psi_{x}(N)-\psi_{x}(N-1) \\
-A^{T} \psi_{x}(N) A
\end{array}\right] x(k+N \mid k)
\end{aligned}
$$

The Proposition 2 assumptions imply:

$$
\begin{aligned}
& \psi_{x}(i)-\psi_{x}(i-1) \\
&= w_{1}(i) Q_{1}+w_{2}(i) Q_{2}-w_{1}(i-1) Q_{1}-w_{2}(i-1) Q_{2} \\
&= {\left[w_{1}(i)-w_{1}(i-1)\right] Q_{1}+\left[w_{2}(i)-w_{2}(i-1)\right] Q_{2} } \\
&= \Delta Q_{1}(i)+\Delta Q_{2}(i) \geq 0 \quad i=1, \cdots, N-1 \\
& \psi_{u}(i)-\psi_{u}(i-1) \\
&=w_{1}(i) R_{1}+w_{2}(i) R_{2}-w_{1}(i-1) R_{1}-w_{2}(i-1) R_{2} \\
&=\left[w_{1}(i)-w_{1}(i-1)\right] R_{1}+\left[w_{2}(i)-w_{2}(i-1)\right] R_{2} \\
&=\Delta R_{1}(i)+\Delta R_{2}(i) \geq 0 \quad i=1, \cdots, N-1 \\
& \psi_{x}(N)-\psi_{x}(N-1)-A^{T} \psi_{x}(N) A \\
&=w_{1}(N) P_{1}-w_{1}(N-1) Q_{1}-w_{1}(N) A^{T} P_{1} A \\
& \quad+w_{2}(N) P_{2}-w_{2}(N-1) Q_{2}-w_{2}(N) A^{T} P_{2} A>0
\end{aligned}
$$

Therefore, $V(x(k))-V(x(k+1))>0$.

Remark 1 . The conditions (11) are for the asymptotic stability of every single general combined MPC. If Algorithm 1 is used to generate weights of these controllers, then the conditions (11) simplify as:

$$
\begin{gathered}
P_{1}-A^{T} P_{1} A-Q_{1}>0, P_{2}-A^{T} P_{2} A-Q_{2}>0 \\
Q_{1} \leq Q_{2}, R_{1} \leq R_{2}
\end{gathered}
$$

The soft switching process actually includes a finite number of small hard switches which constitute a sequential switched system. At each time step during the switching process, a different combined MPC is applied to the plant yielding the corresponding Lyapunov function. Hence, the switching process properties can be investigated based on the multiple Lyapunov functions. In (Branicky, 1998; Liberzon, 2003), it has been argued that for time continuous systems if values of multiple Lyapunov functions of the switched system decrease at the switching points, then the switched system is asymptotically stable. Here, although the above considered soft switching process ends in finite time steps, it is still reasonable to expect the values of multiple Lyapunov functions during the switching process decrease in order to ensure good performance of SS-MPC.

Theorem 1. Consider the switching process under General Combined Method with weighting vectors generated by Algorithm 1. If the conditions (12a) and (12b) are met, then during the switching process the values of multiple Lyapunov functions of SS-MPC strictly decrease.

Proof. As at each $k$ the conditions of Proposition 2 are satisfied (see Remark 1), the general combined MPC is asymptotically stable and the corresponding Lyapunov functions can be defined as:

$$
V(x(k))=\min _{u(\cdot k)} J_{\text {GeneralCombined }}(x(k), u(\cdot \mid k))
$$


At switching time instants the following holds:

$$
\begin{aligned}
V & (x(k)-V(x(k+1)) \\
\geq & x^{T}(k \mid k)\left[w_{1}^{k}(0) Q_{1}+w_{2}^{k}(0) Q_{2}\right] x(k \mid k) \\
& +u_{o p t}^{T}(k \mid k)\left[w_{1}^{k}(0) R_{1}+w_{2}^{k}(0) R_{2}\right] u_{o p t}(k \mid k) \\
& +\sum_{i=1}^{N-1}\left[\begin{array}{l}
x^{T}(k+i \mid k)\left[\left(w_{1}^{k}(i)-w_{1}^{k+1}(i-1)\right) Q_{1}\right. \\
\left.+\left(w_{2}^{k}(i)-w_{2}^{k+1}(i-1)\right) Q_{2}\right] x(k+i \mid k)
\end{array}\right] \\
& +\sum_{i=1}^{N-1}\left[\begin{array}{l}
u_{o p t}^{T}(k+i \mid k)\left[\left(w_{1}^{k}(i)-w_{1}^{k+1}(i-1)\right) R_{1}\right. \\
\left.+\left(w_{2}^{k}(i)-w_{2}^{k+1}(i-1)\right) R_{2}\right] u_{o p t}(k+i \mid k)
\end{array}\right] \\
& +x^{T}(k+N \mid k)\left[w_{1}^{k}(N) P_{1}+w_{2}^{k}(N) P_{2}\right] x(k+N \mid k) \\
& -x^{T}(k+N \mid k)\left[\begin{array}{l}
w_{1}^{k+1}(N-1) Q_{1}+ \\
w_{2}^{k+1}(N-1) Q_{2}
\end{array}\right] x(k+N \mid k) \\
- & x^{T}(k+N \mid k) A^{T}\left[\begin{array}{l}
w_{1}^{k+1}(N) P_{1}+ \\
w_{2}^{k+1}(N) P_{2}
\end{array}\right] A x(k+N \mid k)
\end{aligned}
$$

So, if the following four conditions can be satisfied, it is sufficient to obtain $V(x(k)-V(x(k+1))>0$ :

$$
\begin{aligned}
& {\left[w_{1}^{k}(i)-w_{1}^{k+1}(i-1)\right] Q_{1}+\left[w_{2}^{k}(i)-w_{2}^{k+1}(i-1)\right] Q_{2} \geq 0(13 \mathrm{a})} \\
& {\left[w_{1}^{k}(i)-w_{1}^{k+1}(i-1)\right] R_{1}+\left[w_{2}^{k}(i)-w_{2}^{k+1}(i-1)\right] R_{2} \geq 0(13 \mathrm{~b})} \\
& w_{1}^{k}(N) P_{1}-w_{1}^{k+1}(N-1) Q_{1}-A^{T}\left[w_{1}^{k+1}(N) P_{1}\right] A+w_{2}^{k}(N) P_{2} \\
& -w_{2}^{k+1}(N-1) Q_{2}-A^{T}\left[w_{2}^{k+1}(N) P_{2}\right] A>0
\end{aligned}
$$

where $i=1, \cdots, N-1$. It can be proved from (10a) and (10d) in Algorithm 1 that:

$$
\begin{array}{ll}
w_{1}^{k}(i)-w_{1}^{k+1}(i-1)=0 & i=1, \cdots, N-1 \\
w_{2}^{k}(i)-w_{2}^{k+1}(i-1)=0 & i=1, \cdots, N-1
\end{array}
$$

Therefore, conditions (13a) and (13b) are satisfied. Due to $(10 \mathrm{~b})$ and $(10 \mathrm{c})$ the following holds:

$$
\begin{aligned}
& w_{1}^{k+1}(N)=w_{1}^{k+1}(N-1)=w_{1}^{k}(N) \\
& w_{2}^{k+1}(N)=w_{2}^{k+1}(N-1)=w_{2}^{k}(N)
\end{aligned}
$$

So, $w_{1}^{k}(N) P_{1}-w_{1}^{k+1}(N-1) Q_{1}-A^{T}\left[w_{1}^{k+1}(N) P_{1}\right] A$

$$
+w_{2}^{k}(N) P_{2}-w_{2}^{k+1}(N-1) Q_{2}-A^{T}\left[w_{2}^{k+1}(N) P_{2}\right] A=
$$$$
w_{1}^{k}(N)\left(P_{1}-Q_{1}-A^{T} P_{1} A\right)+w_{2}^{k}(N)\left(P_{2}-Q_{2}-A^{T} P_{2} A\right)
$$

Due to (10b), (10d) and (12a),

$$
w_{1}^{k}(N)\left(P_{1}-Q_{1}-A^{T} P_{1} A\right)+w_{2}^{k}(N)\left(P_{2}-Q_{2}-A^{T} P_{2} A\right)>0
$$

hence, (13c) holds.

\section{NUMERICAL EXAMPLES}

Comparisons between the soft switching methods and the hard switching approach will now be presented by simulation. Consider a second order linear time-invariant system whose continuous-time transfer function is:

$$
H(s)=(s+1) /\left(s^{2}+s+4\right) .
$$

The corresponding discrete time state space model with sampling time 0.2 sec reads:

$$
A=\left[\begin{array}{rr}
0.7496 & -0.7059 \\
0.1765 & 0.9261
\end{array}\right] \quad B=\left[\begin{array}{l}
0.1765 \\
0.0185
\end{array}\right] C=\left[\begin{array}{ll}
1 & 1
\end{array}\right] D=0
$$

The task is to apply a new MPC controller to regulate the system to the origin under the following input constraint after the system has been controlled by an old MPC controller for some period.

$-3.8 \leq u(k) \leq 3.8$

The prediction horizons of the old and the new MPC controllers are both equal to $N=30$.

The control objectives and parameters of the old model predictive controller, named MPC1, are: $Q=1 I, R=0.01 I$ with a selected sufficiently large terminal weight $P=500 I$ to guarantee stability. The final state reached by MPC1 before the switching starts is $[0.0005,0.8623]^{T}$. The new MPC controller, named MPC2, is based on the following parameters as $Q=I, R=10 I$ and $P=500 I$.

The switching process starts at time $k=k_{s}=10$. The switching mechanism parameters of the Convex Combined method and General Combined method are $\alpha=\overline{1: 30} \times 1 / 30$ and $\lambda=0.9$, respectively.

Hard switching, Convex Combined method of soft switching and General Combined method of soft switching respectively takes $0 \mathrm{~s}, 6 \mathrm{~s}$ and $6 \mathrm{~s}$ to fully engage the new controller, and $8.6 \mathrm{~s}, 10.2 \mathrm{~s}$ and $9.4 \mathrm{~s}$ to reach the steady state after the switching process has started.

It can be seen from the simulation results (shown as Fig. 1 - Fig. 6) that both Convex Combined method and General Combined method of soft switching give better transient switching performance in system output and control input than hard switching. However, it takes a little bit more time for the two soft switching methods to fully engage new MPC controller and to reach origin.

\section{CONCLUSIONS}

A dedicated softly switched MPC mechanism with two specific methods to achieve good switching performance has been presented in this paper. Sufficient conditions for asymptotic stability of the proposed SS-MPC have been derived. As only input constraints are considered, the proposed SS-MPC is suitable for systems with not active state constraints. However, if the state constraints are present, designing the soft switching mechanism will greatly complicate. If in addition a feasible set of the new MPC is much smaller than that of the old one, a hard switching may not be feasible at all and the soft switching is then the only switching strategy that is capable of maintaining feasibility of plant operation during the switching process. Design of SS-MPC to cover situations with state constraints is under current research. 


\section{ACKNOWLEDGEMENT}

This work has been supported by Universities UK on behalf of the Department for Education and Skills (DfES) through the UK funding councils and by the Polish State Committee for Scientific Research under grant No.4 T11A 008 25. The authors wish to express their thanks for the support.

\section{REFERENCES}

Bloemen, H., T. Boom and H. Verbruggen (2002). Optimizing the end-point state-weighting matrix in model-based predictive control. Automatica, 38(6), 1061-1068.

Branicky, M.S. (1998). Multiple Lyapunov functions and other analysis tools for switched and hybrid systems. IEEE Transaction on Automatic Control, 43(4), 475 - 482.

Grochowski, M. (2003). Intelligent control of integrated wastewater treatment system under full range of operating conditions. $\mathrm{PhD}$ thesis, Gdansk University of Technology.

Grochowski, M., M. A. Brdys and T. Gmiński (2004a). Intelligent control structure for integrated wastewater systems. Proc. of $10^{\text {th }}$ IFAC/IFORS/IMACS/IFIP Symp. on Large Scale Complex Systems, 1, 263-268, Osaka, July

Grochowski, M., M. A. Brdys, T. Gmiński and P. Deinrych (2004b). Softly switched model predictive control for control of integrated wastewater treatment systems at medium time scale. Proc. of $10^{\text {th }}$ IFAC/IFORS/IMACS/IFIP Symp. on Large Scale Complex Systems, 1, 269274, Osaka, July.

Liberzon, D. (2003). Switching in Systems and Control. Birkhauser, Boston.

Mayne, D.Q., J.B. Rawlings, C.V. Rao and P.O.M. Scokaert (2000). Constrained model predictive control: stability and optimality. Automatica, 36(6), 789-814.

Muske, K.R., and J.B. Rawlings (1993). Model predictive control with linear models. A.I.CH.E Journal, 39(2), 262-297.

Primbs, J.A. and V. Nevistic (2000). A new approach to stability analysis for constrained finite receding horizon control without end constraints. IEEE Transaction on Automatic Control, 45(8), 1507-1512.

Qin, J.S. and T.A. Badgwell (2003). A survey of industrial model predictive control technology. Control Engineering Practice, 11(7), 733-764.

Zheng, A. and M. Morari (1995). Stability of model predictive control with mixed constraints, IEEE Transaction on Automatic Control, 40(10), 1818 $-1823$.

Zheng, A. (1997). Stability of model predictive control with time-varying weights, Journal of Computers \& Chemical Engineering, 21(12), 1389-1393.

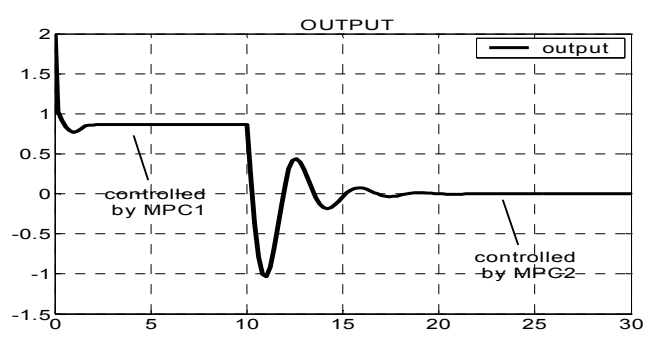

Fig. 1. Hard Switch - output

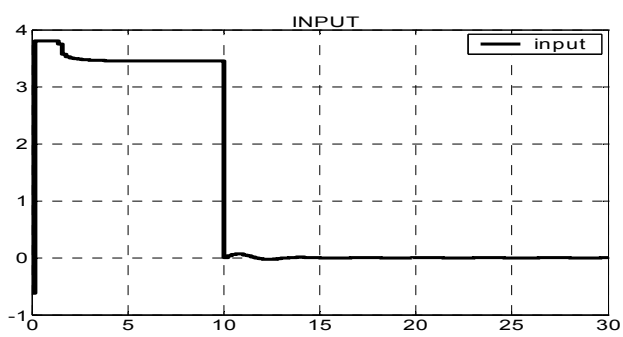

Fig. 2. Hard Switch - control input

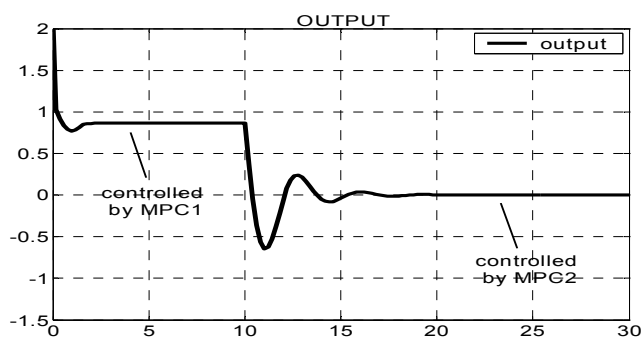

Fig. 3. Convex Combined Method - output

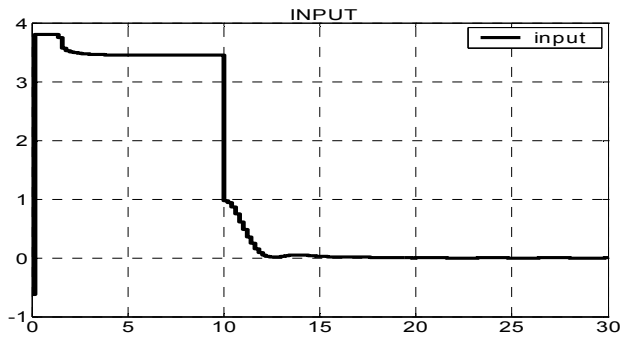

Fig. 4. Convex Combined Method - control input

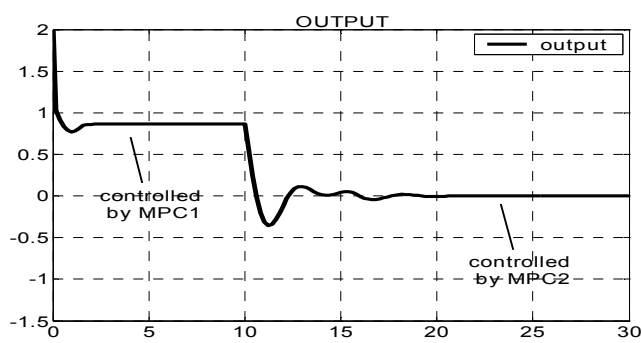

Fig. 5. General Combined Method - output

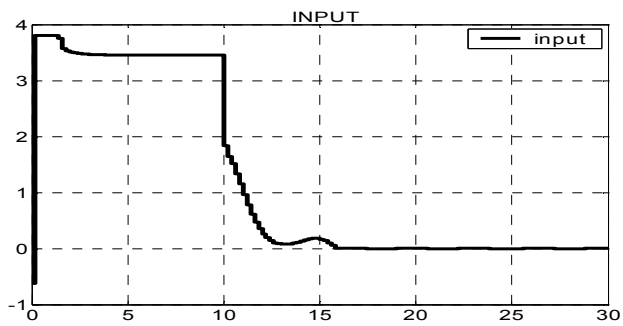

Fig. 6. General Combined Method - control input 